\title{
Relative Ion Yields Measured with a High-Resolution Glow Discharge Mass Spectrometer Operated with an Argon / Hydrogen Mixture
}

\author{
R. W. Smithwick III, D. W. Lynch, and J. C. Franklin \\ Plant Laboratory, Oak Ridge Y-12 Plant, Martin Marictta Energy Systems, Inc. Oak Ridge, Tenuessee, USA
}

Quantitative elemental analysis by glow discharge mass spectrometry (GDMS) requires a calibration factor for each element. The calibration factors used in the present work are called relative ion yields (RIYs). The RIYs of each of 19 elements within samples of four National Institute of Standards and Technology steel reference materials (nos. 661-664) were measured using pure argon and an argon mixture containing $1.0 \%$ hydrogen by volume. The RIYs measured using pure argon correlated within a factor of approximately $2-3$ to the RIYs calculated by a theoretical model. The RIYs measured for these 19 elements using the argon mixture containing $1.0 \%$ hydrogen correlated within a factor of approximately 1.3 to the calculated RTYs. These results may have significant analytical potential with respect to GDMS and may have application to other plasma techniques. (J Am Soc Mass Spectrom 1993, $4,278-285)$

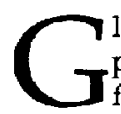
low discharge mass spectrometry (GDMS) is a popular and powerful analytical method $[1,2]$ for determining the elemental composition of metals and other solids. GDMS combines high sensitivity with high dynamic range to measure elemental compositions from the $99+w t \%$ level down to the 1-wt ppm level within approximately a 30-min survey analysis of all the elements. Levels below the 1-wt ppm, and even below the 1-wt ppb level, can be obtained by using a sufficiently long data acquisition time across the peak of each isotope. Mass interferences become more numerous and more critical as smaller peaks are measured to obtain lower detection levels.

A double-sector glow discharge mass spectrometer is capable of both high sensitivity and high resolution. High resolution helps to eliminate peak interferences that, at unit mass resolution, would make unknown contributions to the peak intensities at the nominal masses of the isotopes of the elements being analyzed.

Although the glow discharge phenomenon [3] is complex, a dc glow discharge has a relatively simple electrical and mechanical configuration capable of generating a highly constant source of ions derived from the solid sample. A glow discharge is obtained by applying a dc potential to a metallic sample sur-

Address reprint requests to Robert W. Smithwick III, P.O. Box 2009, Building 9995, MS-8189, Oak Ridge, TN 37831-8189. rounded by argon at a pressure near 1 torr. The potential on the sample (cathode) should be negative (for example, $-1000 \mathrm{~V}$ ) with respect to a second electrode (anode) within the argon. Under conditions similar to these, a visible glow surrounds the sample. Argon ions and other positively charged ions within the plasma are accelerated into the sample by the negative potential, causing atoms to be sputtered from the surface of the sample. These sputtered sample atoms become ionized within the argon plasma by one of several mechanisms, including Penning ionization and electron-impact ionization.

Quantitative determination of the elemental composition of a sample requires a calibration factor for each observed element. In former work [4], calibration factors called relative ion yields (RIYs) were discussed. RIYs are measured by acquiring the mass spectrum of a reference material and measuring the intensities of the isotopes of each of the elements that are certified with respect to composition:

$R \Gamma Y_{\mathrm{s}}=\frac{(\text { wt } \%)_{\mathrm{Fe}}(\text { Isotope area })_{\mathrm{s}} /(\text { Isotope abundance })_{\mathrm{s}}}{(\text { wt \% })_{\mathrm{s}}(\text { Isotope area })_{\mathrm{Fe}} /(\text { Isotope abundance })_{\mathrm{Fe}}}$

For steel samples, the RIY of each element in the sample (s) is computed relative to an isotope of the element iron ( $\mathrm{Fe}$ ) (eq 1). Multiplying both sides of eq 1 by $(w t \%)_{s} / R_{s}$ results in an equation for $(w t \%)_{s}$ that 
is useful for quantitating a sample of unknown composition by assigning values for the RIY $Y_{\mathrm{s}}$ of the elements. In the calculation of the composition of each element, the measured peak area of an isotope is divided by the RTY of that element. If (wt \%) ${ }_{\mathrm{Fe}}$ is not known precisely for the purpose of quantitation, an assumed value can be subsequently scaled to achieve $100 \mathrm{wt} \%$ for the sum of the elements. Relative sensitivity factors (RSFs), also commonly used, are the reciprocals of the RIYs, making them factors that multiply the measured peak areas in the calculation of elemental composition.

Other workers [5] have tabulated experimental values of the RSFs of 56 elements within different sample matrices. After considering numerous factors that could affect the RSFs, these workers chose parameters for (1) Penning ionization, (2) electron-impact ionization, and (3) three-body collisional processes to model RSFs. The RSFs calculated by their model agreed with the measured RSFs within a factor of 2-3 for most, but not all, elements.

In former work [4], the RIYs of 84 elements were calculated by a simple two-parameter theoretical model described by a sputter factor, an ionization factor, and an atomic weight factor. A strong correlation was observed by plotting RTYs calculated by this model with respect to the corresponding RIYs of 17 elements within a Cr-V steel standard [National Institute of Standards and Technology (NIST) 1263a] that was analyzed [6] on a prototype glow discharge quadrupole mass spectrometer. In that work, the worst agreement within this set of 17 elements was a factor of 2 between the experimental and calculated RIYs of sulfur.

Initially, we were unable to measure RIYs for steel standards by GDMS that would correlate to calculated RTYs as well as the measured RIYs of NIST 1263a in ref 6 had correlated. RIYs measured and reported by other researchers did not correlate as well either. The survey mass spectrum of NIST 1263a in ref 6 showed a high ratio (near 10:1) of $\mathrm{ArH}^{+}$to $\mathrm{Ar}^{+}$, indicating that a significant quantity of hydrogen was present within the argon plasma, even though no large water peaks or hydrocarbon peaks were evident within this survey spectrum.

In the present work, both pure argon and a mixture of argon containing $1 \%$ hydrogen by volume were each used to measure the RIYs of 19 certified elements in four NIST steel reference standards (nos. 661-664, set no. S668). The addition of $1 \%$ hydrogen to the argon was found to significantly improve the correlation between the measured RTY's and the RTY's calculated from a simple theoretical model. Mass spectra were acquired with a high-resolution glow discharge mass spectrometer.

\section{Experimental}

The high-resolution glow discharge mass spectrometer used in this work was a Concept S32 manufactured by Kratos Analytical, Ltd. (Ramsey, NJ). It is a double- sector instrument of Nier-Johnson geometry. This instrument was supplied with a Sun-3E computer (Sun Microsystems, Inc., Mountain View, CA) having a 32bit processor capable of 3 million instructions per second. This computer has 16 Mbytes of main memory and 380 Mbytes of memory on a hard disk. The computer software is accessed through a windows menu to perform such operations as data acquisition, mass calibration, and the calculation of elemental composition.

Survey data obtained by scanning the magnetic field can be mass calibrated (by assignments of known peaks) to high accuracy over a mass range, 2-300 $u$, sufficient for GDMS. The combination of high resolution and high accuracy permits centroided peaks to be assigned reliably to the isotope ions of the elements or to molecular ions. (Molecular ions are proposed by the operator within a software file.) Any portion of a mass spectrum may be displayed, with the observed peaks labeled as calibrated mass, area, or symbol (of atomic or molecular ion). These labels are assigned automatically to the mass-calibrated spectrum by the criterion of matching masses within a specified mass tolerance (of $100 \mathrm{ppm}$, for example).

Several additions and modifications have been made to the instrument. To measure the pressure within the source, the glow cell was connected by a 0.1-in. i.d. tube to an MKS Baratron pressure transducer. Because the source operates at a potential of $+8000 \mathrm{~V}$ and because argon at a pressure near 1 torr becomes electrically conductive between gradients of high voltage, the pressure head and digital readout were isolated with a transformer and operated at $+8000 \mathrm{~V}$ above the electrical ground of the instrument. The transformer, the pressure gauge, a high-voltage power supply, and several digital voltage displays were enclosed within a clear plastic box having 0.5 -in.-thick walls. With a Baratron reading of 1.2 torr in the glow cell, the ion gauge pressure reading within the electrostatic analyzer remained less than $5 \times 10^{-8}$ torr. These pressure readings are displayed continuously while the instrument is in full operation.

The argon gas inlet line was coiled around a cold finger containing liquid nitrogen to condense water and hydrocarbon impurities out of the argon before the gas enters the glow cell. Because argon has a boiling point that is $10{ }^{\circ} \mathrm{C}$ above the boiling point of nitrogen, the argon gas must be at a pressure sufficiently below atmospheric pressure to prevent liquidizing the argon by the liquid nitrogen.

Samples of four NIST steel reference materials (nos. 661-664, set no. S668) were analyzed separately in 99.997\% pure argon and in an argon mixture containing $1.0 \%$ hydrogen by volume. Each analysis was initiated with a fresh end of the appropriate reference material. Glow discharge mass spectra were obtained by using a gas pressure reading of 1.20 torr and $a$ cathode-anode voltage reading of $-1000 \mathrm{~V}$. The peak corresponding to ${ }^{54} \mathrm{Fe}$ (which has a natural abundance of 5.8 atom \%) was scaled to approximately $9 \mathrm{~V}$ (of the 


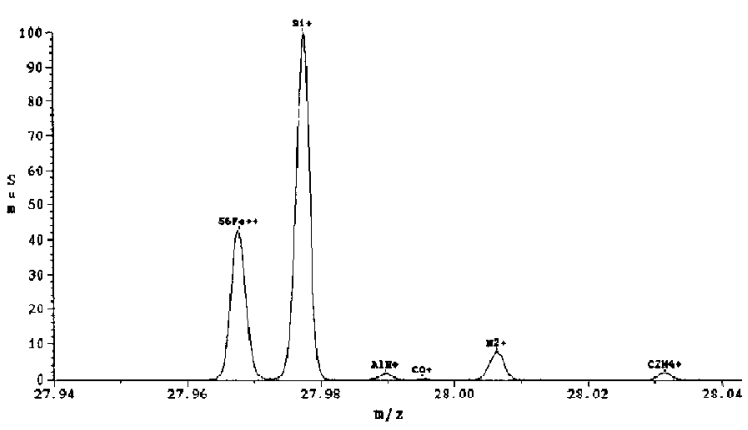

Figure 1. High-resolution glow discharge mass spectrum of the region near $m / z 28$ obtained for NIST steel reference material no. 664. The resolution is approximately 6000 .

$10-\mathrm{V}$ amplifier) by adjusting the gain of the electron multiplier. The peak corresponding to ${ }^{56} \mathrm{Fe}$ is off scale. A postacceleration detector was the only type of detector used in the present work.

Mass spectra were scanned from 5 to $250 \mathrm{u}$ in a survey mode under DAC-scan mode with magnetic field control. This scan range involved over one-half million time channels. The scan rate was set to $60 \mathrm{~s}$ per decade, and the mass resolution was set near 7000 (peak width definition at $5 \%$ of peak height). The Hall probe was maintained at a temperature constant to within $\pm 0.01{ }^{\circ} \mathrm{C}$ by using a Neslab Model-RTE refrigerated, recirculating water bath.

For each separate analysis, approximately 2 Mbytes of data were stored on disk within an arquisition time of $1 \mathrm{~h}$. Data acquisition for each analysis was started within 5-10 min after the glow discharge had been initiated on a fresh sample. Eighteen scans during the last $30 \mathrm{~min}$ of each analysis were summed and then mass calibrated.

Figure 1 shows a 0.1 mass width of the mass spectrum of NIST no. 664 near $m / z 28$. The tallest peak is derived from the $660 \mathrm{wt}$ ppm of silicon in this steel reference standard. The resolution of the peaks within the spectrum in Figure 1 was approximately 6000, indicating a high degree of instrument stability for this operational mode over a 30-min time period. Figure 1 demonstrates that this resolution is adequate to separate multicharged ion interferences and hydride ion interferences.

\section{Results, Calculations, and Discussion}

The RIYs measured for the 19 major elements in NIST standard steel materials (nos. 661-664) are shown in Table 1 for pure argon and for an argon mixture containing $1.0 \%$ hydrogen. Each isotope of a given element permits an independent determination of the RIY of that element if the peak corresponding to that isotope gives an accurately measured area and if the peak is predominantly free of mass interference. In addition to the RIYs shown in Table 1, 14 other determinations of the RIYs of minor isotopes (C, Si, S, Ti, $\mathrm{Cr}, \mathrm{Fe}, \mathrm{Ni}$, and $\mathrm{Mo}$ ) were also measured. The peak corresponding to ${ }^{54} \mathrm{Fe}$ is an example of a peak that is predominantly free of mass interference. It is an important example because all of the measured RTY's in the present work are referenced to ${ }^{54} \mathrm{Fe}$. Although ${ }^{54} \mathrm{Cr}$ is a mass interference to ${ }^{54} \mathrm{Fe}$ (when the mass spectrometer has a resolution below approximately 80,000), no more than approximately $0.5 \%$ of the total area assigned to ${ }^{54} \mathrm{Fe}$ is contributed by ${ }^{54} \mathrm{Cr}$ in the steel reference standards analyzed in the present work. For this reason, the area of the peak has been assigned exclusively to ${ }^{54} \mathrm{Fe}$ without correcting for the ${ }^{54} \mathrm{Cr}$. By contrast, ${ }^{54} \mathrm{Cr}$ has an enormous interference from ${ }^{54} \mathrm{Fe}$.

An average value for the measured RIY of each of the 19 elements in Table 1 was computed in the following manner (for pure argon and separately for argon containing $1 \%$ hydrogen). In the case of RIYs measured for a single isotope of an element, a simple average was taken among the measurements of the different steel standards. In the case of multiple isotopes having multiple values of RIY for the same element, an average RIY was first computed for the element by weighing the measured RIY of each isotope by its natural abundance divided by the total abundances of the isotopes involved in the average; then the average was taken among the measurements of the different steel samples. For an individual analysis, this definition is equivalent to calculating RIY by first summing all of the interference-free areas associated with a given element and dividing by the sum of the abundances associated with that total area. In addition to the RIYs in Table 1, 14 other RIYs of minor isotopes were also used to compute average RIYs. The average measured RIY for 19 elements is shown in Table 2 for pure argon and for argon containing $1 \%$ hydrogen.

The following equation was used for the purpose of calculating RIYs for each of 96 elements from a theoretical model:

$\mathrm{RTY}_{\mathrm{s}}=$

(Mass factor) ${ }_{\mathrm{s}}$ (Ionization factor $)_{\mathrm{s}}$ (Atomic weight $)_{\mathrm{Fe}}$ ${\text { (Mass factor) }{ }_{\mathrm{Fe}} \text { (Ionization factor) }{ }_{\mathrm{Fe}} \text { (Atomic weight) }}_{\mathrm{s}}$

The atomic weight factors in eq 2 are necessary to convert from a per-atom basis to a per-gram basis.

In the present work, an empirical mass-dependent factor was substituted for the sputter factor used in former work [4]: 
Table 1. Individual values of RIYs for isotopes of 19 elements measured for NIST steel sample nos. 661-664 with pure argon and with argon containing $1 \%$ hydrogen by volume

\begin{tabular}{|c|c|c|c|c|c|c|c|c|c|}
\hline $\begin{array}{l}\text { Obs } \\
\text { Ion }\end{array}$ & $\begin{array}{l}\text { Percent } \\
\text { Isotope } \\
\end{array}$ & $\begin{array}{c}\text { RIYs } \\
\text { No. 661 } \\
\text { Argon }\end{array}$ & $\begin{array}{c}\text { RIYs } \\
\text { No. } 662 \\
\text { Atgon }\end{array}$ & $\begin{array}{c}\text { RIYs } \\
\text { No. } 663 \\
\text { Argon }\end{array}$ & $\begin{array}{c}\text { RIYs } \\
\text { No. } 664 \\
\text { Argon }\end{array}$ & $\begin{array}{c}\text { RIYs } \\
\text { No. } 661 \\
1 \% \mathbf{H}_{2}\end{array}$ & $\begin{array}{c}\text { RIYs } \\
\text { No. } 662 \\
1 \% \mathrm{H}_{2}\end{array}$ & $\begin{array}{c}\text { RIYs } \\
\text { No. } 663 \\
1 \% \mathrm{H}_{2}\end{array}$ & $\begin{array}{c}\text { RIYs } \\
\text { No. } 664 \\
1 \% \mathrm{H}_{2} \\
\end{array}$ \\
\hline${ }^{12} \mathrm{C}$ & 98.89 & 0.350 & 0.617 & 0.328 & 0.356 & 0.195 & 0.188 & 0.144 & 0.156 \\
\hline${ }^{27} \mathrm{Al}$ & 10000 & - & 1.315 & 1.057 & - & 2.538 & 1.675 & 2.156 & - \\
\hline${ }^{\mathrm{x}} \mathrm{Si}$ & 92.21 & 0.646 & 0.745 & 0.656 & 0.680 & 0.991 & 0.962 & 0.956 & 0.987 \\
\hline${ }^{31} \mathrm{P}$ & 100.00 & 0.366 & 0.408 & 0.414 & 0.301 & 0.216 & 0.228 & 0.217 & 0.170 \\
\hline${ }^{n} \mathrm{~S}$ & 95.00 & 0.445 & 0.500 & 0.625 & 0.447 & 0.209 & 0.168 & 0.215 & 0.161 \\
\hline${ }^{\infty} \mathrm{Ti}$ & 73.94 & 2.853 & 3.256 & 2.924 & 2.939 & 1.586 & 1.850 & 1.684 & 1.635 \\
\hline siv & 99.74 & 1.967 & 1.742 & 1.860 & 1.819 & 1.357 & 1.172 & 1.258 & 1.313 \\
\hline${ }^{32} \mathrm{Cr}$ & 83.76 & 0.550 & 0.618 & 0.523 & 0.572 & 1.102 & 1.108 & 1.106 & 1.183 \\
\hline${ }^{*} \mathrm{Mn}$ & 100.00 & 0.689 & 0.713 & 0.660 & 0.650 & 1.072 & 1.082 & 1.064 & 1.067 \\
\hline${ }^{\mathbf{s}_{4}} \mathrm{Fe}$ & 5.82 & 1.000 & 1.000 & 1.000 & 1.000 & 1.000 & 1.000 & 1.000 & 1.000 \\
\hline${ }^{59} \mathrm{Co}$ & 100.00 & 0,922 & 0,998 & 0.887 & 0.977 & 0.798 & 0.775 & $0.73 ?$ & 0.793 \\
\hline${ }^{80} \mathrm{Ni}$ & 26.0) & 0.629 & 0.630 & 0.618 & 0.597 & 0.711 & 0.692 & 0.710 & 0.705 \\
\hline${ }^{s} \mathrm{Cu}$ & 69.09 & 0.215 & 0.238 & 0.204 & 0.222 & 0.520 & 0.514 & 0.530 & 0.559 \\
\hline${ }^{6} \mathrm{Cu}$ & 30.91 & 0.185 & 0.241 & 0.207 & 0.204 & 0.510 & 0.514 & 0.487 & 0.533 \\
\hline${ }^{75} \mathrm{As}$ & 100,00 & 0.271 & 0.266 & 0.253 & 0.224 & 0.202 & 0.166 & 0.162 & 0.151 \\
\hline${ }^{90} \mathrm{Zr}$ & 51.46 & 1.597 & 1.691 & 1.612 & 1.693 & 0.649 & 0.771 & 0.832 & 0.815 \\
\hline${ }^{91} \mathrm{Zr}$ & 11.23 & 1.074 & 1.676 & 1.475 & 1.600 & 0.622 & 0.788 & 0.812 & 0.770 \\
\hline${ }^{93} \mathrm{Nb}$ & 100.00 & 1.641 & 1.597 & 1.661 & 1.576 & 0.657 & 0.700 & 0.766 & 0.718 \\
\hline${ }^{*} \mathrm{Mo}$ & 23.78 & 0.995 & 0.980 & 0.852 & 0.973 & 0.828 & 0.730 & 0.750 & 0.777 \\
\hline${ }^{\mathrm{I}} \mathrm{T} \mathrm{Ta}$ & 99.99 & 0.747 & 0.656 & 0.756 & 0.730 & 0.290 & 0.304 & 0.330 & 0.331 \\
\hline${ }^{185} \mathrm{~W}$ & 26.41 & 0.650 & 0.739 & 0.671 & 0.725 & 0.292 & 0.347 & 0.320 & 0.312 \\
\hline${ }^{183} \mathrm{~W}$ & 14.40 & 0.534 & 0.708 & 0.654 & 0.675 & 0.245 & 0.334 & 0.263 & 0.290 \\
\hline${ }^{184} w$ & 30.64 & 0.622 & 0.732 & 0.668 & 0.690 & 0.280 & 0.336 & 0.297 & 0.301 \\
\hline${ }^{186} \mathrm{~W}$ & 28.41 & 0.594 & 0.743 & 0.708 & 0.709 & 0.2860 & 0.325 & 0.308 & 0.227 \\
\hline
\end{tabular}

Table 2. Average values of RIYs for 18 elements measured with pure argon and with argon containing $1 \%$ hydrogen

\begin{tabular}{ccc|ccc}
\hline & \multicolumn{2}{c|}{ Average measured RIY } & & \multicolumn{2}{c}{ Avcrage measured RIY } \\
\cline { 2 - 3 } Element & No hydrogen & 1\% hydrogen & Element & No hydrogen & 1\% hydrogen \\
\hline \hline C & 0.412 & 0.170 & $\mathrm{Co}$ & 0.946 & 0.774 \\
$\mathrm{Al}$ & 1.186 & 2.123 & $\mathrm{Ni}$ & 0.611 & 0.697 \\
$\mathrm{Si}$ & 0.679 & 0.971 & $\mathrm{Cu}$ & 0.217 & 0.524 \\
$\mathrm{P}$ & 0.372 & 0.208 & $\mathrm{As}$ & 0.254 & 0.170 \\
$\mathrm{~S}$ & 0.505 & 0.186 & $\mathrm{Zr}$ & 1.614 & 0.763 \\
$\mathrm{Ti}$ & 2.973 & 1.680 & $\mathrm{Nb}$ & 1.619 & 0.710 \\
$\mathrm{~V}$ & 1.847 & 1.275 & $\mathrm{Mo}$ & 0.924 & $\mathbf{0 . 7 5 0}$ \\
$\mathrm{Cr}$ & 0.564 & 1.124 & $\mathrm{Ta}$ & 0.722 & 0.314 \\
$\mathrm{Mn}$ & 0.678 & 1.071 & $\mathrm{~W}$ & 0.681 & 0.299 \\
\hline
\end{tabular}


There are two reasons for changing from the sputter factor to this mass-dependent factor. One reason is that researchers in GDMS commonly regard the process of sputtering as being mass independent because atoms of an element that sputter with low efficiency are concentrated at the surface. After a few atomic layers have been removed by sputtering, a steady-state rate of sputtering is expected without a mass dependence.

The second reason for using eq 3 is that it is simpler to use than the former equations. A single parameter $B$, taken to be $0.04 / \mathrm{g}$ in the present work, is assumed to determine the shape of the mass dependence regardless of the origin of this mass dependence, and $W_{\mathrm{s}}$ is the average atomic weight of element "s." The term shape is used because multiplying eq 3 by any constant gives the same calculated RIYs, as this constant would cancel within eq 2 because the ratio of each element is taken with respect to the element iron.

The mass dependence of the electron yields that result from ions striking a metal target has been described [7] by equations that are similar to the sputtering factor used in former work [4]. In the present work, this metal target is the aluminum knob of the postacceleration detector (at $-8000 \mathrm{~V}$ ) in front of the electron multiplier. As pointed out in Section 5.3 of ref 5 , the response of an ion-counting detector should be independent of the mass of the ion that strikes the detector, whereas an analog detector (as used in the present work) would have mass dependence. The type of detection used will influence both the measured RTYs and the mass-dependent factor of eq 3 required to best correlate measured and calculated RIYs. Apart from the detector, the mass spectrometer may influence the mass distribution of the ions reaching the detector. It should be mentioned that the ion transmission characteristics of a magnetic sector mass spectrometer show less mass discrimination than those of a quadrupole mass spectrometer.

The ionization factor, used previously, is most easily described as a statistical mechanical concept called an occupation number [8] corresponding to FermiDirac statistics for an ideal gas $[8,9]$ having the following equilibrium process: $\mathbf{M}^{-} \leftrightarrows \mathbf{M}^{+}+2 \mathbf{e}^{-}$. This factor can also be derived by writing the equilibrium constant for the same process in terms of partition functions, followed by some simplifying assumptions [4]:

$$
\text { (Ionization factor) })_{\mathrm{s}}=\frac{1}{1+e^{+11,600\left(\mathrm{EA}_{\mathrm{s}}+\mathrm{IP}_{\mathrm{s}}-\mu\right) / T}}
$$

The constant 11,600 in eq 4 is the reciprocal of the Boltzmann constant and has units of degrees Kclvin per electron volt; $\mathbf{I P}_{\mathrm{s}}$ is the first ionization potential [10], in electron volts; and $\mathbf{E A}_{\mathbf{s}}$ is the electron affinity [11], in electron volts, for a given element. Missing values among the heavy elements within these references were assumed to be $0 \mathrm{eV}$ for electron affinities or $6 \mathrm{eV}$ for ionization potentials. In the present work, the plasma temperature $T$ is taken to be $14,000 \mathrm{~K}(16,000 \mathrm{~K}$ was used in ref 4 ), whereas the chemical potential $\mu$ is taken to be $9.0 \mathrm{eV}$. An element is calculated by eq 4 to be $50 \%$ ionized when $\mathrm{EA}_{\mathrm{s}}+\mathrm{IP}_{\mathrm{s}}$ equals $\mu$.

The RTYs calculated using eqs $2-4$ are shown in Table 3 for 96 elements. The ionization potentials and electron affinities used in this calculation are tabulated in addition to the percentage of positive ions (calculated by eq 4) for each element. The calculated RIYs in Table 3 are similar to those calculated previously for elements having a mass lower than approximately 70 u, but the calculated RIYs for significantly heavier elements are approximately one-half the values of the RTY's calculated previously [4]. The calculated RTYs are intended to represent elements that are sputtered off the surface of the sample, rather than elements that are volatilized from the inner volume of a (porous) sample.

Figure 2 shows the linear plot of the calculated RIYs of Table 3 as a function of the average RIYs of Table 2 for the same element measured in pure argon. The agreement in Figure 2 between measured and calculated RTYs is within a factor of approximately $2-3$. This agreement is comparable to that achieved by others [5] using another theoretical model. If all RTY's were assumed to have the value 1.0 , the agreement with these measured values would be within a factor of 5 .

Figure 3 shows the linear plot of the calculated RIYs of Table 3 as a function of the average RIYs for the same element in Table 2 measured in argon containing $1.0 \%$ hydrogen. The measured RIYs correlate within a factor of approximately 1.3 to the RTYs calculated for these elements. The improved correlation found in Figure 3, compared with Figure 2, may indicate that hydrogen species within the argon plasma cause the populations of sample ions to conform more closely to the thermodynamic function of eq 4. Future work will be required to determine the mechanisms that may give rise to this improved correlation.

The compositions of four rare earth elements were also determined using the data taken with argon containing $1 \%$ hydrogen and using the calculated RIYs from Table 3. These compositions are shown in Table 4 with the certified compositions of NIST steel reference materials nos. 661-663. Although these rare earth elements are present at only a few weight parts per million, the agreement was favorable for this survey analysis.

The temperature $(14,000 \mathrm{~K})$ and the chemical potential $(9.0 \mathrm{eV})$ should be regarded as mathematical parameters until they can be measured within the mass spectrometer. These parameters, which are similar to those discussed and chosen in past work [4], plus the mass-dependent factor $\left(B=0.04 \mathrm{~g}^{-1}\right)$, were chosen in the present work to optimize the agreement shown in Figure 3. Other combinations of closely similar parameters might also have given agreement similar to that shown in Figure 3. Changing these parameters could also change the correlation shown in Figure 2; how- 
Table 3. RTY calculated by eqs $2-4$ for 96 elements using $T=14,000 \mathrm{~K}, \mu=9.0 \mathrm{eV}$, and $B=0.04 \mathrm{~g}^{-1}$

\begin{tabular}{|c|c|c|c|c|c|c|c|c|c|c|c|}
\hline \multicolumn{2}{|c|}{ Element } & \multirow{2}{*}{$\frac{\text { IP }}{13.60}$} & \multirow{2}{*}{$\frac{\mathrm{EA}}{0.77}$} & \multirow{2}{*}{$\frac{\%+\text { lon }}{1.16}$} & \multirow{2}{*}{$\begin{array}{c}\text { RIY } \\
0.04\end{array}$} & \multicolumn{2}{|c|}{ Element } & \multirow{2}{*}{$\frac{\mathrm{IP}}{5.78}$} & \multirow{2}{*}{$\begin{array}{l}\text { EA } \\
0.20\end{array}$} & \multirow{2}{*}{$\%+$ Ion } & \multirow{2}{*}{$\begin{array}{l}\text { RIY } \\
0.78 \\
\end{array}$} \\
\hline 1 & $\mathrm{H}$ & & & & & 49 & In & & & & \\
\hline 2 & $\mathrm{He}$ & 24.48 & -0.22 & 0.00 & 0.00 & so & Sn & 7.34 & 1.03 & 62.76 & 0.52 \\
\hline 3 & $\mathrm{Li}$ & 5.39 & 0.62 & 92.25 & 3.17 & 51 & Sb & 8.64 & 0.94 & 38.21 & 0.31 \\
\hline 4 & $\mathrm{Bre}$ & 9.32 & 0.38 & 35.89 & 1.19 & 52 & Te & 9.01 & 1.96 & 16.35 & 0.13 \\
\hline 5 & B & 8.30 & 0.18 & 60.61 & 1.94 & 53 & 1 & 10.45 & 3.06 & 2.33 & 0.02 \\
\hline 6 & C & 11.26 & 1.29 & 5.01 & 0.16 & 54 & $\mathrm{Xe}$ & 12.13 & -0.45 & 9.79 & 0.07 \\
\hline 7 & $\mathbf{N}$ & 14.53 & -0.21 & 1.20 & 0.04 & 55 & $\mathrm{Cs}$ & 3.89 & 0.39 & 98.04 & n.72 \\
\hline 8 & 0 & 13.61 & 1.46 & 0.65 & 0.02 & 56 & $\mathrm{Ba}$ & 5.21 & -0.48 & 97.17 & 0.69 \\
\hline 9 & $F$ & 17.42 & 3.50 & 0.01 & 0.00 & 57 & La & 5.61 & 0.55 & 91.32 & 0.64 \\
\hline 10 & $\mathrm{Ne}$ & 21.56 & -0.30 & 0.00 & 0.00 & 58 & $\mathrm{Ce}$ & 5.60 & 0.60 & 91.05 & 0.64 \\
\hline 11 & $\mathbf{N a}$ & 5.14 & 0.54 & 94.00 & 2.42 & 59 & $\mathrm{Pr}$ & 5.46 & 0.30 & 93.61 & 0.65 \\
\hline 12 & $\mathbf{M g}$ & 7.65 & 0.22 & 78.60 & 1.98 & 60 & No & 5.51 & 0.10 & 94.32 & 0.64 \\
\hline 13 & A & 5.98 & 0.20 & 91.19 & 2.20 & 61 & $\mathrm{Pm}$ & 5.50 & 0.00 & 94.78 & 0.64 \\
\hline 14 & $\mathbf{S i}$ & 8.15 & 1.36 & 39.59 & 0.94 & 62 & $\mathrm{Sm}$ & 5.60 & 0.00 & 94,36 & 0.62 \\
\hline 15 & $\mathbf{P}$ & 10.48 & 0.71 & 14.01 & 0.32 & 63 & $\mathrm{Eu}$ & 5.67 & 0.00 & 94.04 & 0.61 \\
\hline 16 & $\mathbf{s}$ & 10.36 & 2.04 & 5.64 & 0.13 & 64 & $\mathrm{Gd}$ & 6.16 & 0.20 & 89.91 & 0.56 \\
\hline 17 & $\mathrm{Cl}$ & 13.01 & 3.62 & 0.18 & 0,00 & 65 & $\mathrm{~Tb}$ & 5.98 & 0.00 & 92.43 & 0.57 \\
\hline 18 & Ar & 15.76 & -0.37 & 0.50 & 0.01 & 66 & Dy & 6.80 & 0.00 & 86.09 & 0.52 \\
\hline 19 & $\mathrm{k}$ & 4.34 & 0.47 & 96.99 & 1.93 & 67 & Ho & 6.00 & 0.00 & 92.31 & 0.55 \\
\hline 20 & Ca & 6.11 & -1.93 & 98.19 & 1.93 & 68 & $\mathrm{Er}$ & 6.08 & 0.00 & 91.83 & 0.54 \\
\hline 21 & sc & 6.54 & -0.73 & 93.36 & 1.71 & 69 & Trm & 5.81 & 0.00 & 93.36 & 0.54 \\
\hline 22 & $\mathrm{Ti}$ & 6.82 & -0.02 & 86.09 & 1.51 & 70 & $\mathrm{Yb}_{\mathrm{b}}$ & 6.20 & 0.00 & 91.05 & 0.52 \\
\hline 23 & $\mathrm{v}$ & 6.74 & 0.63 & 79,42 & 1.33 & 71 & Lu & 6.00 & 0,00 & 92.31 & 0.52 \\
\hline 24 & $\mathrm{Cr}$ & 6.76 & 0.97 & 74.12 & 1.23 & 72 & Hf & 7.00 & -0.63 & 89.84 & 0.50 \\
\hline 25 & Mn & 7.43 & -0.97 & 89.13 & 1.42 & 73 & Ta & 7.88 & 0.15 & 69.08 & 0.38 \\
\hline 26 & $\mathrm{Fe}$ & 7.87 & 0.46 & 63.53 & 1.00 & 74 & $\mathbf{w}$ & 7.98 & 1.23 & 45.65 & 0.24 \\
\hline 27 & $\mathrm{Co}_{0}$ & 7.86 & 1.06 & 51.66 & 0.78 & 75 & $\mathrm{Re}$ & 7.87 & 0.38 & 65.05 & 0.34 \\
\hline 28 & $\mathbf{N i}$ & 7.63 & 1,62 & 44.84 & 0.68 & 76 & $O_{6}$ & 8.50 & 1.44 & 31.46 & 0.16 \\
\hline 29 & $\mathrm{Cu}$ & 7.72 & 1.80 & 39.39 & 0.56 & 77 & Ir & 9.00 & 1.97 & 16.35 & 0.08 \\
\hline 30 & Zn & 9.39 & 0.09 & 40.19 & 0.56 & 78 & $\mathrm{Pt}$ & 9.00 & 2.56 & 10.71 & 0.05 \\
\hline 31 & Ga & 6.00 & 0.37 & 89.84 & 1.19 & 79 & Au & 9.22 & 2.80 & 7.57 & 0.04 \\
\hline 32 & Ge & 7.88 & 1.44 & 43.41 & 0.56 & 80 & $\mathrm{Hg}$ & 10.43 & -0.19 & 26.36 & 0.13 \\
\hline 33 & As & 9.81 & 1.07 & 17.40 & 0.22 & 81 & T1 & 6.11 & 0.32 & 89.37 & 0.43 \\
\hline 34 & Se & 9.75 & 2.12 & 8.49 & 0.10 & 82 & $\mathrm{~Pb}$ & 7.42 & 1.03 & 61.20 & 0.29 \\
\hline 35 & $\mathrm{Br}$ & 11.84 & 3.36 & 0.58 & 0.01 & 83 & $\mathbf{B i}$ & 7.29 & 0.95 & 65.24 & 0.31 \\
\hline 36 & $\mathbf{K r}_{\mathbf{r}}$ & 14.00 & -0.42 & 220 & 0.02 & 84 & Po & 8.43 & 1.32 & 34.95 & 0.16 \\
\hline 37 & $\mathbf{R b}$ & 4.18 & 0.42 & 97.46 & 1.09 & 85 & At & 9.50 & 0,00 & 39.79 & 0.19 \\
\hline 38 & $\mathbf{S r}$ & 5.69 & -1.51 & 98.19 & 1.07 & 86 & $R_{n}$ & 10.75 & 0.00 & 19.00 & 0.08 \\
\hline 39 & $\mathbf{Y}$ & 6.38 & 0.40 & 9243 & 0.99 & 87 & $\mathrm{Fr}$ & 4.00 & 0.00 & 98.44 & 0.43 \\
\hline 40 & $\mathrm{Z}_{\boldsymbol{r}}$ & 6.84 & 0.45 & 200.48 & 0.85 & 88 & $\mathbf{R}_{a}$ & 5.28 & 0.00 & 95.62 & 0.42 \\
\hline 41 & Nb & 6.88 & 1.13 & 69.43 & 0.72 & 89 & Ac & 6.90 & 0.000 & 85.07 & 0.37 \\
\hline$\$ 2$ & Mo & 7.10 & 1.18 & 64.49 & 0.65 & 90 & $\mathrm{Th}$ & 6.95 & 0.00 & 84.53 & 0.36 \\
\hline 43 & $\mathrm{Tr}$ & 7.28 & 0.99 & 64.68 & 0.64 & 91 & $\mathbf{P a}$ & 6.00 & 0.00 & 92.31 & 0.39 \\
\hline 44 & Ru & 7.36 & 1.51 & 5269 & 0.50 & 92 & $\mathbf{U}$ & 6.08 & 0.00 & 97.83 & A.38 \\
\hline 45 & $\mathbf{R h}$ & 7.46 & 1.68 & 47.10 & 0.44 & 93 & $\mathrm{~Np}$ & 6.80 & 0.00 & 92.31 & 0.38 \\
\hline 46 & Pd & 8.33 & 1.02 & 4280 & 0.39 & 94 & $\mathbf{P u}$ & 5.10 & 0,00 & 96.20 & 0.39 \\
\hline 47 & $\mathrm{Ag}_{\mathrm{g}}$ & 7.57 & 2.00 & 38.41 & 0.35 & 95 & Am & 6.00 & 0.00 & 92.31 & 0.37 \\
\hline 48 & $\mathrm{Cd}$ & 8.99 & -0.27 & 55.77 & 0.48 & 96 & $\mathrm{Cm}$ & 6.00 & 0.00 & 92.31 & 0.37 \\
\hline
\end{tabular}




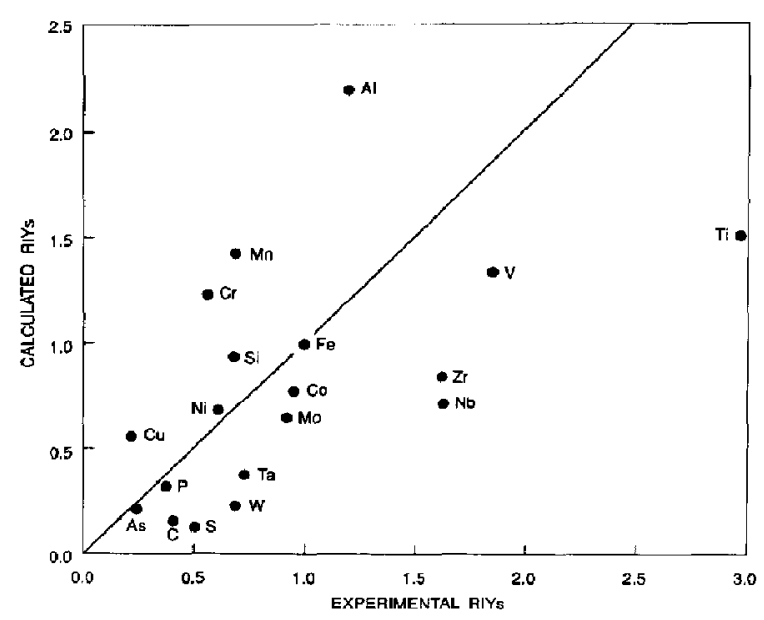

Figure 2. Plot of the calculated RIYs from Table 3 versus the average meastured RYYs from Table 2 that were obtained for samples of NIST steel reference materials (nos. 661-664) using pure argon.

ever, no parameter set was found that would significantly improve the overall correlation represented in Figure 2.

Oxygen, fluorine, and gold are especially interesting because the RIYs calculated for these elements are low, owing to their ionization potentials and positive electron affinities. The RIYs of these elements were not measured in the present work. The calculated RIY of oxygen is shown to be 0.02 in Table 3 . This value corresponds to an RSF of approximately 50, which agrees favorably with the RSF of oxygen measured [5] to be $50 \pm 10$ in steel standards. The calculated RIY of fluorine, shown to be 0.00 in Table 3 , is 0.00014 (corresponding to an RSF of roughly 7000), but no experimental value for fluorine was determined in ref 5 . The

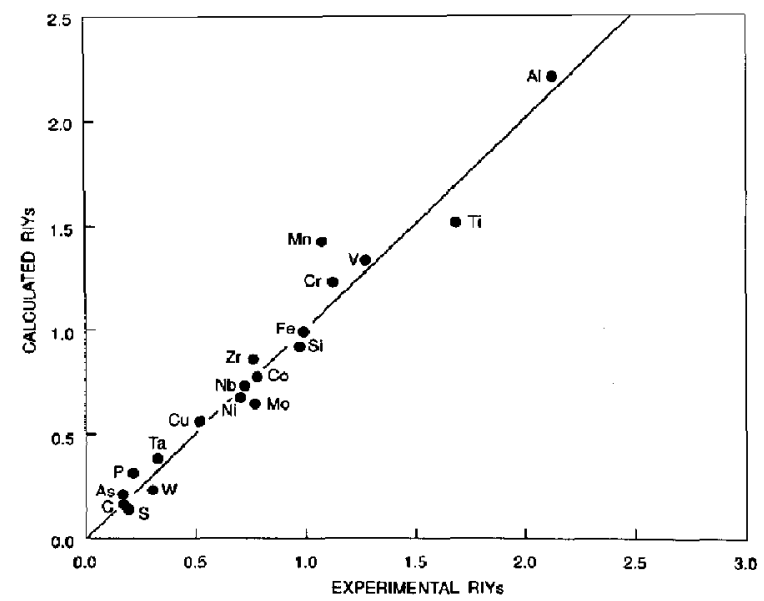

Figure 3. Plot of the calculated RIY's from Table 3 versus the measured RIYs from Table 2 that were obtained for samples of NIST steel reference materials (nos. 661-664) using an argon mixture containing $1 \%$ hydrogen by volume.
Table 4. Comparison of measured and certified compositions of rare earth elements at low-weight parts per million levels

\begin{tabular}{crrrrrrr}
\hline \multirow{2}{*}{$\begin{array}{c}\text { Rare earth } \\
\text { isotope }\end{array}$} & \multicolumn{3}{c}{ Measured composition } & \multicolumn{3}{c}{ Certified composition } \\
\cline { 2 - 4 } & 661 & 662 & 663 & & 661 & 662 & 663 \\
\hline \hline${ }^{199} \mathrm{La}$ & 3 & 3 & 5 & & 4 & 4 & 6 \\
${ }^{140} \mathrm{Ce}$ & 15 & 15 & 12 & & 13 & 11 & 16 \\
${ }^{141} \mathrm{Pr}$ & 1 & 1 & 1 & & 1.4 & 1.2 & 1.8 \\
${ }^{144} \mathrm{Nd}$ & 3 & 3 & 5 & 3 & 5 & 7 \\
\hline
\end{tabular}

calculated RIY of gold is shown in Table 3 to be 0.04, corresponding to an RSF of approximately 25; however, the experimental RSF of gold in a copper matrix is reported [5] to be 2.6. As demonstrated in Figures 2 and 3, the RIY's of some elements change significantly on addition of hydrogen to the argon. Additional work will be required to investigate both a wider variety of elements and a variety of matrices.

In preliminary work, argon containing $0.1 \%$ hydrogen resulted in measured RTYs that were nearly the average of the RIYs measured in pure argon and the RIYs measured in argon containing $1.0 \%$ hydrogen. The RIYs measured in argon containing $10 \%$ hydrogen were roughly the same as those measured in argon containing $1 \%$ hydrogen, except that the measured RIY of phosphorus was reduced by a factor of $2 / 3$, and the measured RIY of sulfur was reduced by a factor of $1 / 2$.

\section{Summary}

RIYs have been measured for samples of NIST steel standards (nos. 661-664) with a high-resolution GDMS operated using pure argon and separately using an argon mixture containing $1.0 \%$ hydrogen by volume. The presence of hydrogen improved the correlation to within a factor of approximately 1.3 between experimental and calculated RIYs. No mechanism was proposed in the present work to explain this improved correlation. The mass dependence, previously assigned to the sputtering phenomenon [4], is now believed to be due mostly to the mass dependence of the electronmultiplier detector operated in analog mode.

The strong correlation between measured and calculated RTYs may have significant analytical potential. Although adding hydrogen to the argon promotes the production of hydrogen-containing ions within the glow discharge, these ions are easily resolved from analyte ions by using a double-sector instrument. Further work is needed to demonstrate its range of application with respect to additional elements and to additional types of samples. Finally, the methods and correlations described in the present work may find application to other plasma techniques.

\section{Acknowledgments}

The Oak Ridge Y-12 Plant is managed for the U.S. Department of Energy by Martin Marietta Energy Systems, Inc., under contract $D E-A C 05-84 O R 21400$. The authors thank Johnie Brown and $Q$. 
Grindstaff for their technical advice and consultation and Tom Harmon, Kathy Cole, and Lynn Marshall for their suggestions and help concerning modifications to the instrument.

\section{References}

1. Harrison, W. W.; Barshick, C. M.; Klinger, J. A.; Ratcliff, P. H.; Mei, Y. Anal. Chem. 1990, 62, 943A-949A.

2. Harrison, W. W.; Hess, K. R.; Marcus, R. K.; King, F. L. Anal. Chem. 1986, 58, 341 A-356A.

3. Chapman, B. Glow Discharge Processes; Wiley: New York, 1980.
4. Smithwick, R. W. III. J. Am. Soc. Mass Spectrom. 1992, 3, 79-84.

5. Vieth, W.; Huneke, J. C. Spectrochim. Acta 1991, 46B, 137-153.

6. Taylor, W. S.; Dulak, J. K. Spectroscopy 1989, 4, 41-46.

7. Beuhler, R. J.; Friedman, L. J. Appl. Phys. 1977, 48, 3928-3936.

8. Andrews, F. C. Equilibrium Statistical Mechanics; Wiley: New York, 1963.

9. Rice, O. K. Statistical Mechanics, Thermodynamics, and Kinetics; W. H. Freeman: San Francisco, 1967.

10. Weast, R. C., Ed. The Hundbook of Chemistry and Plysics, 57th ed.; CRC Press: Cleveland, OH; 1976-1977; p. E-68.

11. Zollweg, R. J. J. Chem. Phys. 1969, 50, 4251-4261. 